\title{
Marine Environment's Effect on the Deterioration of Archaeological Buildings in the Mediterranean Sea Coast (Alexandria - Egypt as an Example)
}

\author{
Rabea Radi Abdel Kader, Shaimaa Sayed Mohamed El-Sayed*, El-Ghareeb Sonbol \\ Restoration Department, Faculty of Archaeology, Cairo University, Cairo, Egypt
}

Email address:

Egyptianconservators2013@gmail.com (S. S. M. El-Sayed)

${ }^{*}$ Corresponding author

To cite this article:

Rabea Radi Abdel Kader, Shaimaa Sayed Mohamed El-Sayed, El-Ghareeb Sonbol. Marine Environment's Effect on the Deterioration of Archaeological Buildings in the Mediterranean Sea Coast (Alexandria - Egypt as an Example). International Journal of Archaeology. Vol. 5, No. 2, 2017, pp. 14-18. doi: 10.11648/j.ija.20170502.11

Received: February 21, 2017; Accepted: April 5, 2017; Published: November 1, 2017

\begin{abstract}
The environment has many severe effects on the Archaeological sites all over the world, it varies from country to the other, there are agriculture, desert and marine environments, the monument is neonate its environment, from the first, the building materials were from the environment, also the deterioration factors are from the environment, this research sheds the light on the marine environment's effect on the deterioration of archaeological sites in the Mediterranean sea coast ( Alexandria - Egypt as an example), the effects vary from the relative humidity, air temperature variations, wind, sea levels rise, underground water, air pollution, rain and tides. The case study tombs in Alexandria which suffer from the marine environment's effect are Kom El - Shoqafa catacombs and El-Shattbi catacombs, the deterioration factors related to the marine environment cause the degradation of these catacombs and this precious cultural heritage.
\end{abstract}

Keywords: Marine Environment, Deterioration, Archaeological Buildings, Mediterranean Sea Coast, Alexandria, Kom El- Shoqafa and El- Shattbi Catacombs

\section{Introduction}

Alexandria is located in the north of Egypt on the Mediterranean Sea coast, its space reaches to $2879 \mathrm{Km}^{2}$, Alexandria has morphological determinants, from the north, there is the Mediterranean sea and Maryot Lake in the south, it characterizes by its climate - (figure 1), the case study catacombs in Alexandria are Kom El- Shoqafa and El Shattbi.

Kom El - Shoqafa catacombs return to the second century $\mathrm{AD}$, they characterize by their wonderful decorations which collect the Egyptian and Greco - Roman ornaments, the catacombs consist of three floors, the first one is full of underground water, the catacombs include also the main well, Rotunda well, the Festivals hall, statues and the burial chambers - ( figure 2).

El- Shabtti catacombs return to the end of Ptolemaic period, there were discovered in 1906, they consist of five funeral buildings which characterize by their decorations and ornaments and they reflect the ancients imaging of the other world - figure 3 .

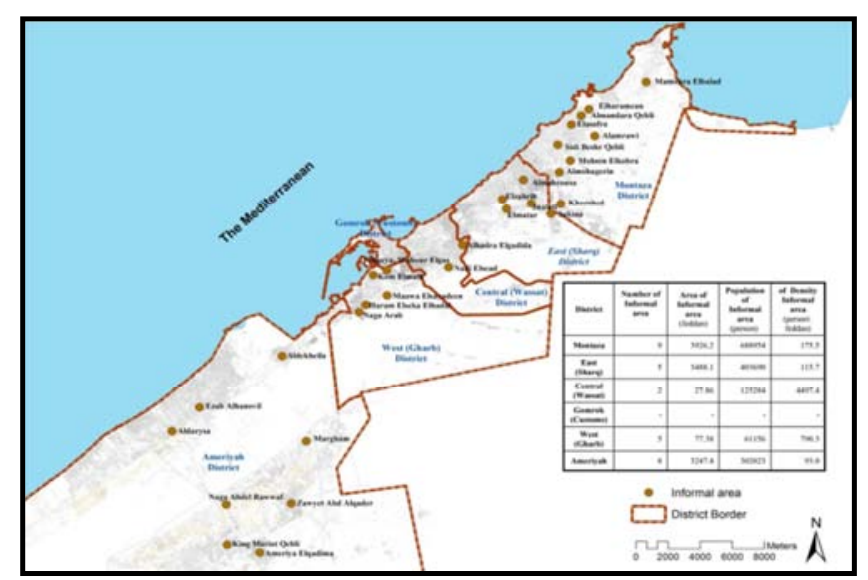

Figure 1. Shows Alexandria location on the Mediterranean Sea. 


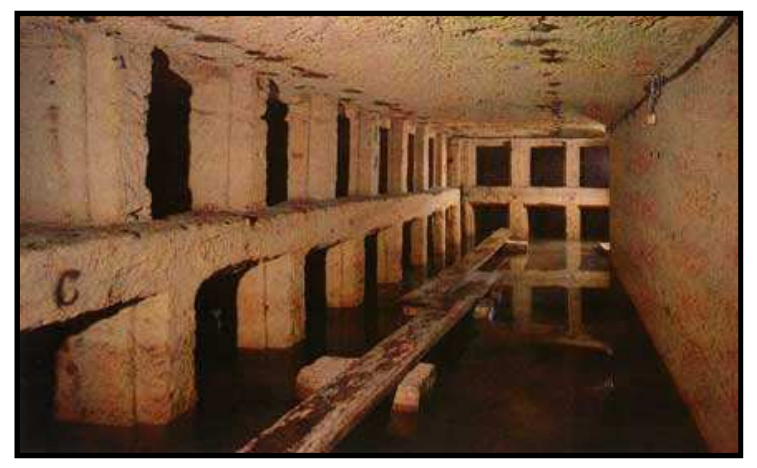

Figure 2. Shows Kom El-Shoqafa catacombs in Alexandria.

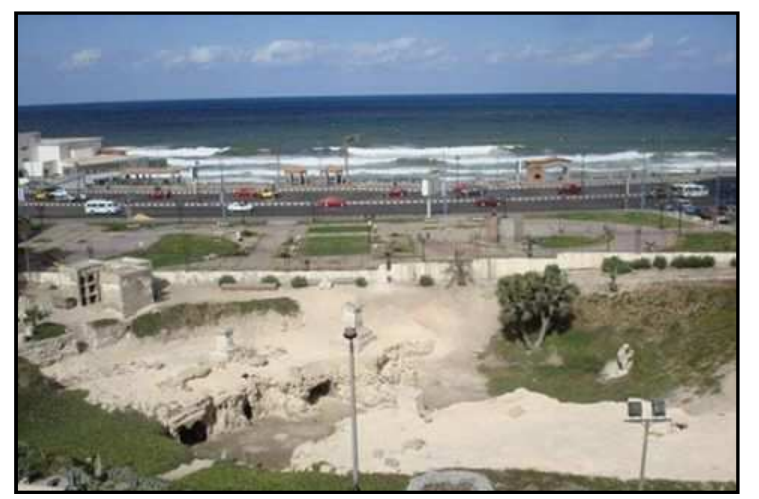

Figure 3. Shows El-Shattbi catacombs location in Alexandria near the sea.

\section{Methodology}

To study the marine environment's effect on the deterioration of archaeological buildings in Alexandria, we have to study the environmental effects all the year like: relative humidity, air temperature variations, rain, wind directions and erosion, underground water, sea levels rise, air pollution and tides, from these studies we can make a vision about the deterioration phenomena causes in the case study catacombs as the following

\subsection{Relative Humidity}

Relative humidity in Alexandria is high in the summer due to the evaporation processes caused by high air temperature degrees and wind, the average relative humidity degrees ranges from $65-70 \%$ - figure 4 , the rising of relative humidity levels is very dangerous to the monuments because it helps in the deterioration with many deterioration factors like: salts, air pollution and microorganisms growth, the result will be the loss of this valuable cultural heritage.

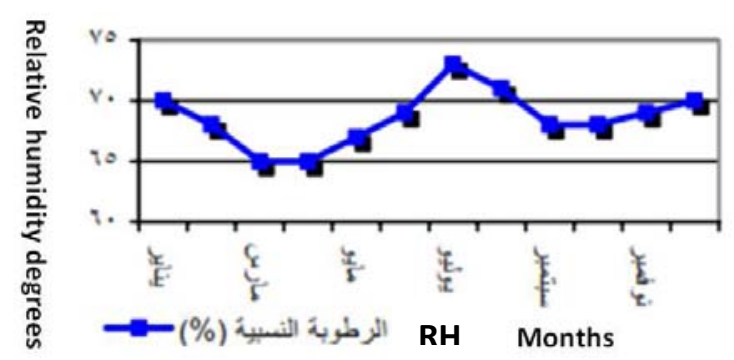

Figure 4. Shows the relative humidity degrees in Alexandria during 2008.

\subsection{Air Temperature Variations}

There is diversity in the air temperature degrees in Alexandria, in the winter; they reach to $13.6^{\circ} \mathrm{C}$ in January, slow rising in February and March till August which they reach to $26^{\circ} \mathrm{C}$ and return to decrease again - (figure5), the rising of air temperature levels will help in the crystallization of salts, insects growth in the archaeological sites and being good reagent in the chemical reactions especially the conversion of air pollution' gases to destructive acids. On the other hand, the low levels of air temperature will help in the microorganisms' growth.

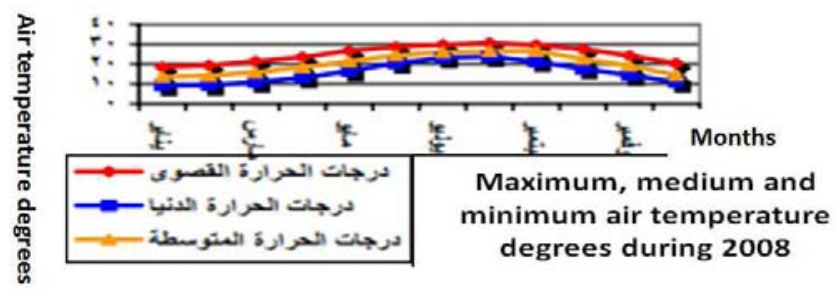

Figure 5. Shows the air temperature degrees in Alexandria during 2008.

\subsection{Rain Rates}

Alexandria distinguishes with the high rates of the rain in Egypt, they reaches to $64.6 \mathrm{~mm}$ in the winter and this rate is high comparing to the other governorates in Egypt, the high rain levels are a good source of humidity in archaeological site, it mean, there is a good environment to the microorganisms growth and air pollution' gases conversion to acids.

\subsection{Wind}

The dominant wind directions in Alexandria are the east, northwest and northeast, the annual rate of the wind's speed is $14.4 \mathrm{Km}$ / hour and reaches to $50 \mathrm{Km} /$ hour in the rough waters season from May to November - (figure 6), wind's high speed means an erosion of the archaeological sites building materials like: lime stone and fired bricks, wind will carry seeds of the wild plants and some suspended materials like sand and dust.

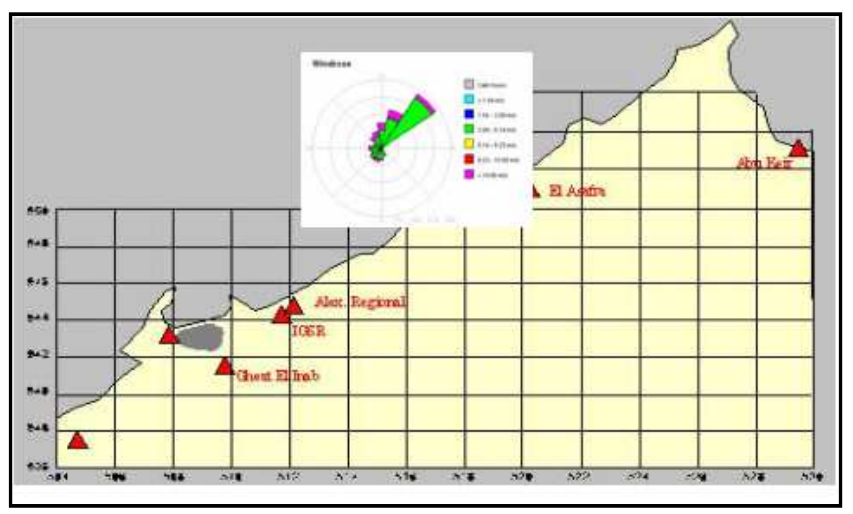

Figure 6. Shows the wind distribution in Alexandria during May 2006.

\subsection{Underground Water}

Alexandria distinguishes with high levels of underground 
water with an acceptable salinity above the saline water, the existence of some archaeological catacombs in low levels making the groundwater a big problem in Alexandria beside the rising of sea level, the problem is in the soil, foundations, architectural elements and building materials.

\subsection{Tide's Phenomenon}

Tide is considered one of the cosmic natural phenomena which happens due to the incidence of seas and oceans under two forces, the first one is the gravity emerging from the Moon and the second one is the centrifugal force resulted from the Earth's rotation around itself, the result is a horizontal movement of the water masses on the Earth near to or far from the coast line, this phenomenon reaches its top in seas and oceans near to the equator, it reaches in Alexandria' ports to $0.46 \mathrm{~m}$ above the fixed levels of the map.

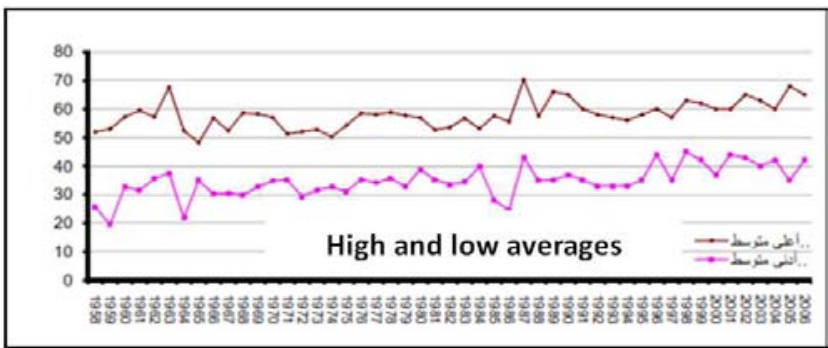

Figure 7. Shows the sea levels from 1958-2006 in Alexandria.

\subsection{The Changes of Sea Levels}

The Rising of sea levels is considered one of the important problems in the world due to the global warming phenomenon and the melting of ice, this melting rises the water levels in the seas, Alexandria suffers from this phenomenon due to its location on the Mediterranean sea coast, this phenomenon reflects on the deterioration of the case study catacombs because they locate in low levels comparing to the sea level in Alexandria, there is wobbling direction between the increase and the decrease of sea levels in Alexandria through 49 years ( from 1958 -2006), its reaches its maximum value in 1987 (57.02) and reaches its minimum value in 1972 (40.73) - (figure 7-8).

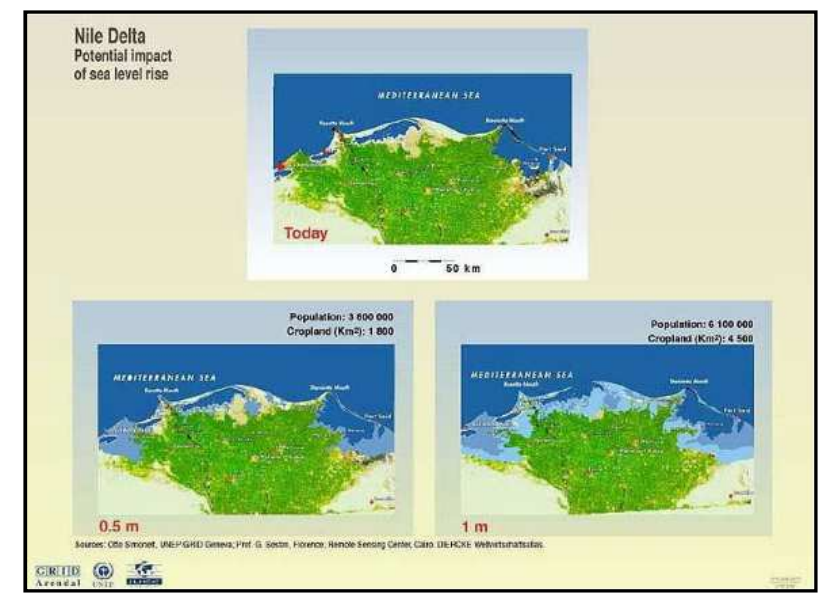

Figure 8. Shows the potential sea levels rising in the future.

\subsection{Air Pollution}

The Air pollution in Alexandria results from some industries like: cement, carbon black's industry, the using of diesel in the running of some factories beside the cars wastes, there are some efforts to improve the type of the air from Alexandria governorate and the Environmental Affairs System in Cairo (The Ministry of Environment), the problem is the conversion of air pollution' gases to dangerous destructive gases like: sulphuric dioxide conversion to sulphuric acid in the existence of high humidity levels, this acid reacts with limestone( calcium carbonates - calcite $\mathrm{CaCO}_{3}$ ) and converts to (calcium sulphates $\mathrm{CaSO}_{4}$ ), the result is the loss of the building materials in the end because calcium sulphates is more soluble than calcium carbonates.

\subsection{The Marine Environment's Effect on the Deterioration of the Case Study Catacombs in Alexandria}

\subsubsection{Kom El-Shoqafa Catacombs}

The rising of sea levels in Alexandria and the underground water are the big problem in these catacombs because they are in a low level comparing the surrounding area, the sea water and the underground water gather in the first floor of these catacombs and they are good sources for the algae growth and the crystallization of salts on the limestone (the building material), these algae secret organic acids which react with calcium carbonates ( the main component of limestone) and convert it to water-soluble materials, also the water erodes the stone by the time and affects badly on the soil and foundations of the tomb.

Salts are one of the big problems resulted from the existence of sea water, it raises by the capillary system of the stone and salts crystallize under the stone surface or effloresce on the surface, they grow in the pores of the stone and press on the sides of these pores, the result is the stone crumbling or stone bleeding. Air pollution gases also are one of the problems due to the plenty of humidity in the catacombs to convert them to dangerous acids reacting with the stone and severely deteriorate it - (figure 9:12).

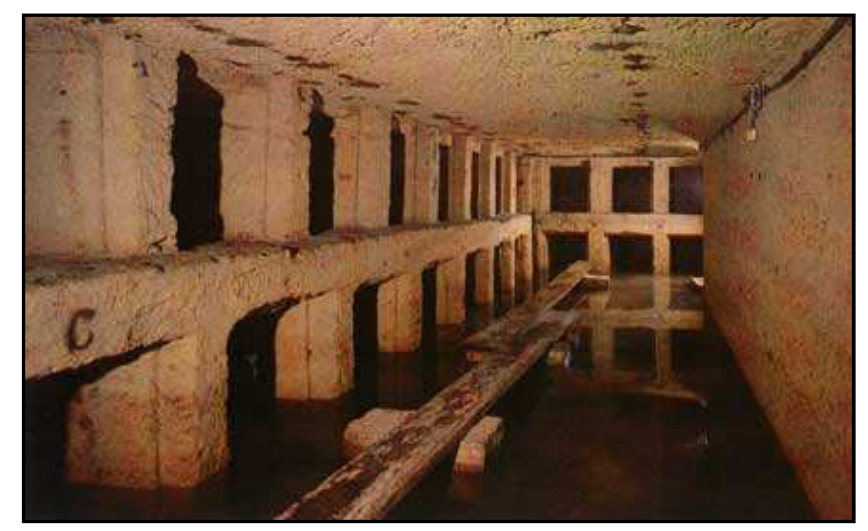

Figure 9. Shows the high levels of water in the first floor of Kom El-Shoqafa catacombs. 


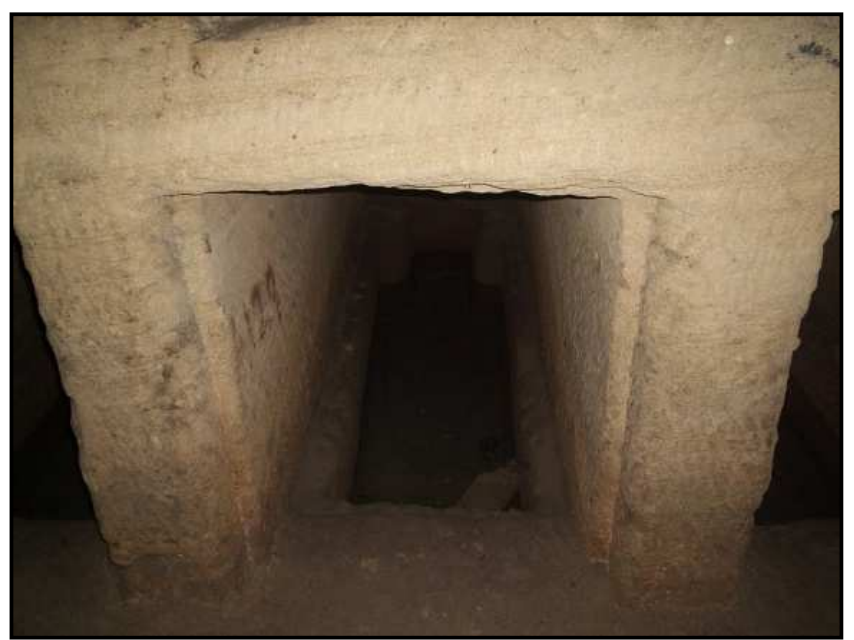

Figure 10. Also shows the high levels of water in one of the first floor tombs in Kom El-Shoqafa catacombs.

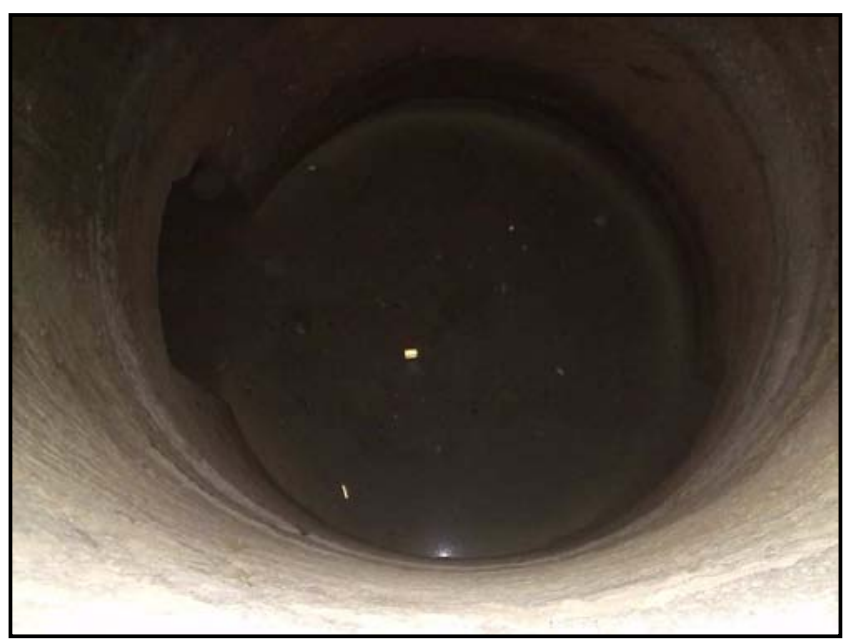

Figure 11. Shows the filling of the catacombs main well with water.

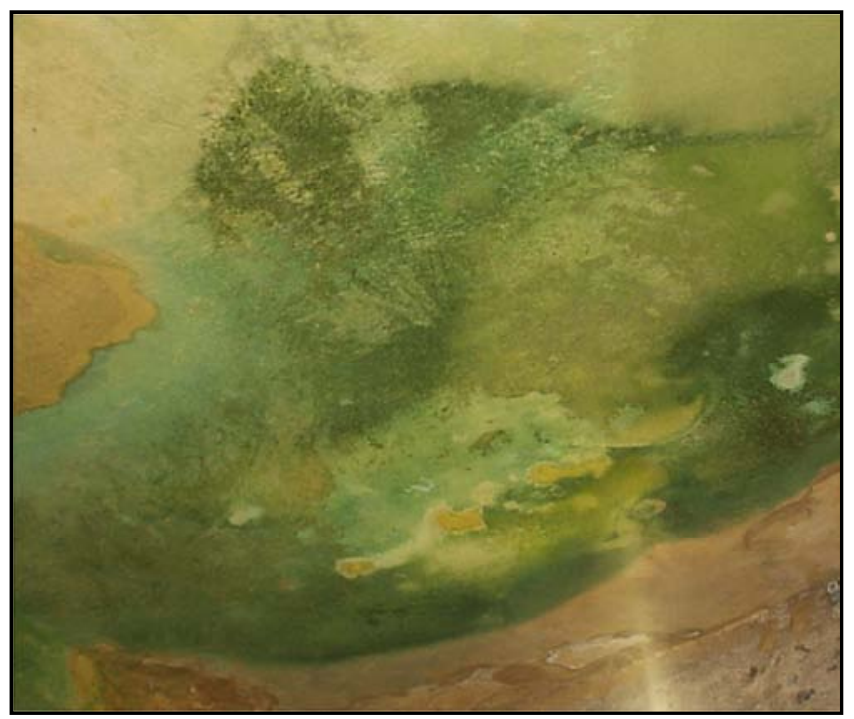

Figure 12. Shows the algae growth on lime stone in Kom El-Shoqafa catacombs.

\subsubsection{El-Shattbi Catacombs}

These catacombs suffer from the rising of sea levels because they are very near to the sea and in a low level comparing to the surrounding area, this case is a very suitable environment for the microorganisms' growth like: algae, also the salts growth and air pollution gases conversion. The wind erosion is very clear in the catacombs facades, water existence in high levels in the tombs affects on their soil and foundations -(figure 13:17).

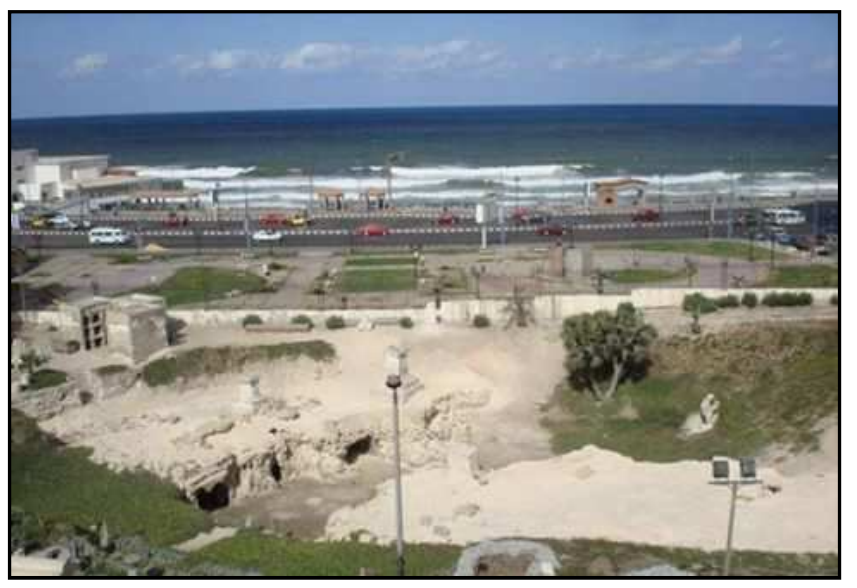

Figure 13. Shows El-Shattbi catacombs and their approach to the sea and the street.

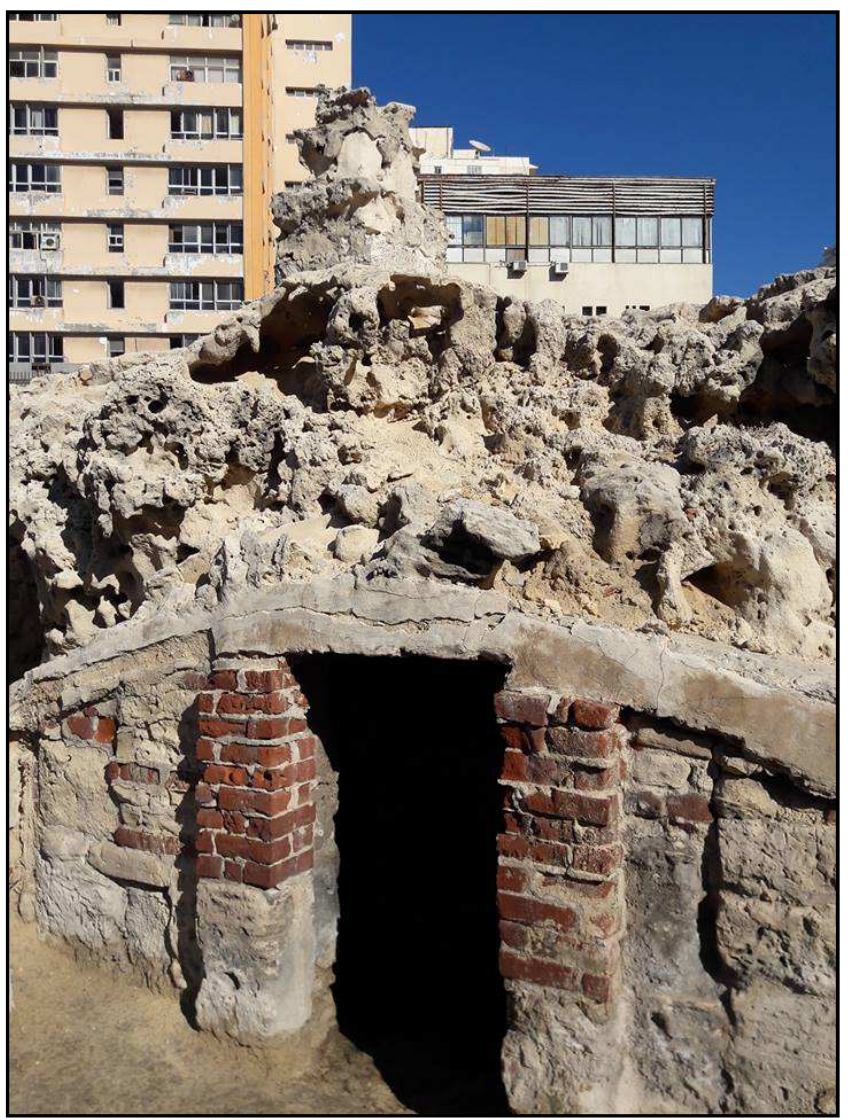

Figure 14. Shows wind erosion of one of the catacombs' facades. 


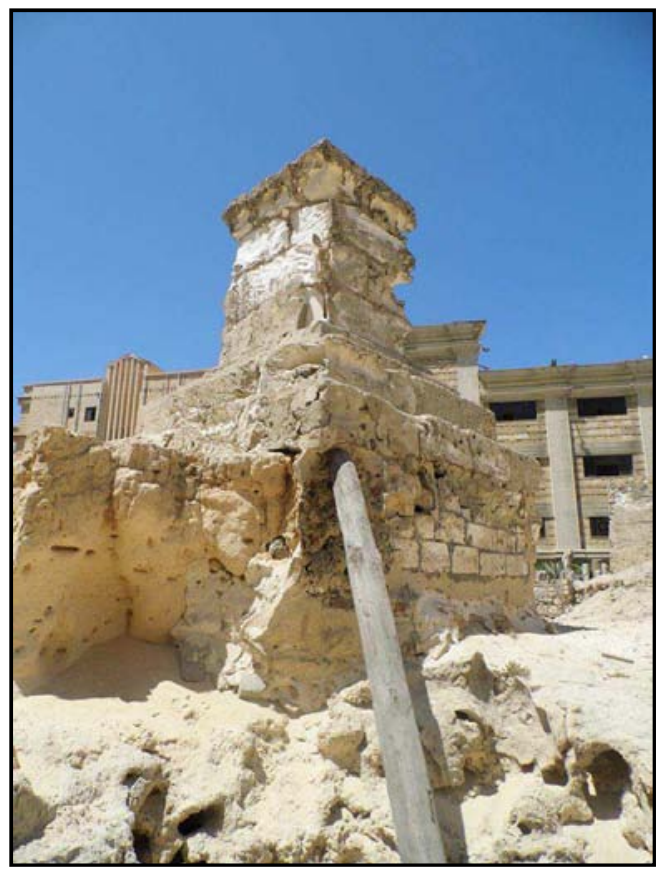

Figure 15. Also shows the wind erosion of the building materials (Fired brick and Lime stone) in the catacombs facades.

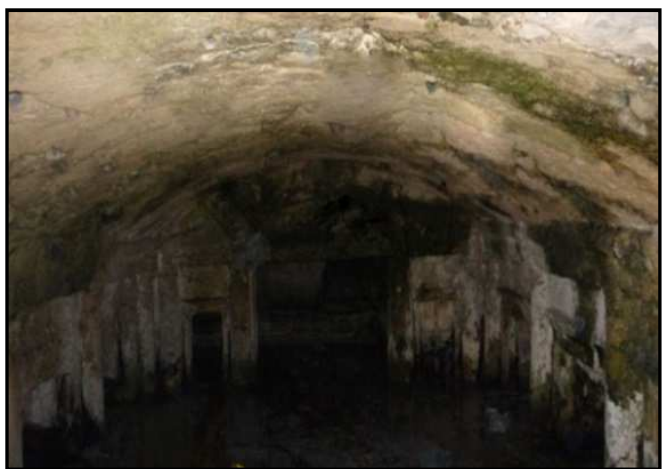

Figure 16. Shows the high growth of algae on the building materials.

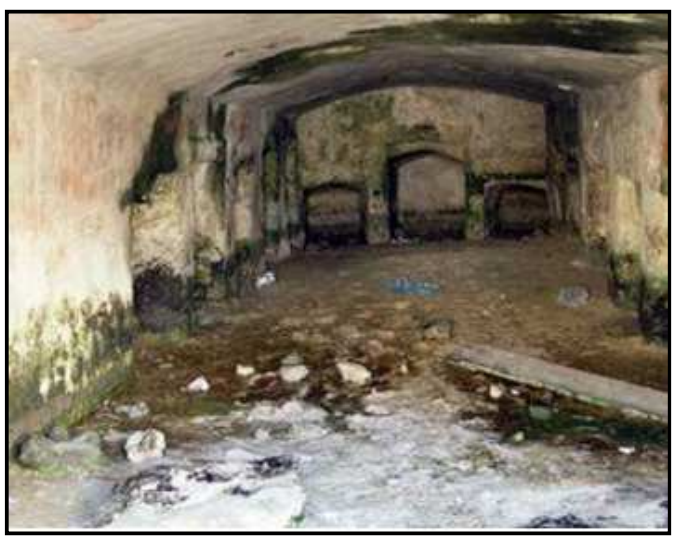

Figure 17. Shows the high levels of water inside the catacombs and also algae growth on the building materials.

\section{Discussion}

The Marine Environment in Alexandria affects badly on the archaeological building, the environmental effects like: relative humidity, air temperature variations, rising of sea levels, wind, air pollution, tides and under groundwater play a great role in the deterioration of the case study catacombs, by studying these environmental determinants and their effect on the archaeological building deteriorations, we can make a vision about this problem and put a tight plan for their restoration and conservation for the upcoming generations.

\section{Conclusion}

The monument is neonate its environment, its building materials were from its environment and the deterioration factors also from this environment, there are many affecting environments: agricultural, desert and marine environments, each one affects with a different way from the others, the Environmental determinants differs in each environment and these determinants are deterioration factors for the building materials and sites all over the world.

\section{References}

[1] The Ministry of Housing, Utilities and Urban Development., "Prepare a Comprehensive Overview of The Housing and to Monitor Changes in The Housing Market Issues (Alexandria city)", Egypt, January 2010, PP: 14-15.

[2] Environmental affairs system (Ministry of Environment), Alexandria Governorate., "Environmental Description of Alexandria ", Egypt, 2007, PP: 8-9.

[3] The Ministry of Housing, Utilities and Urban Development., "The Environmental Perspective of The Strategy of Urban Development on The Level of the Republic", Alexandria Region, PP:86-96.

[4] Environmental affairs system (Ministry of Environment)., " The Monthly Report of Air Type in Egypt", Egypt, May 2006, P:20.

[5] Shaimaa Sayed Mohamed El- Sayed Mahgoub, Study of the burial environment effect on the deterioration of the excavated Alabaster Objects, Restoration and Conservation methods applied on some Chosen Objects, MSc thesis, Restoration department, Faculty of Archaeology, Cairo University,Cairo Egypt, 2012.

[6] Shaimaa Sayed Mohamed El- Sayed Mahgoub, Evaluation of Wild and Domestic Trees and Plants Hazards, their Role in the Deterioration of Archaeological Buildings Ruins, Methods of Treatment and Assessment of these Hazards Applied on a Chosen Historical Building and Site, PhD thesis, Restoration department, Faculty of Archaeology, Cairo University,Cairo Egypt, 2015/2016.

[7] Rabea Radi Abdel Kader Sayed, A scientific study to evaluate the materials which used as alternative support for Greco Roman mosaics to choose the best for the application on a chosen object, MSc thesis, Restoration department, Faculty of Archaeology, Cairo University,Cairo - Egypt, 2015/2016.

[8] Rabea Radi Abdel Kader and Shaimaa Sayed Mohamed, "The study of ground water effect and risks on the safety of the Archaeological Islamic buildings in Egypt applied on Azabk El- Youssefi Madrasa", Built Heritage 2013 Monitoring Conservation Management conference, Milan - Italy, 2013, PP: 1212-1216. 